\title{
Anatomic Single Bundle Anterior Cruciate Ligament Reconstruction by Low Accessory Anteromedial Portal Technique: An In Vivo 3D CT Study
}

\author{
Kwang Won Lee, MD, Yoon Sub Hwang, MD, Yong Joo Chi, MD, Dae Suk Yang, MD, Ha Yong Kim, MD, and \\ Won Sik Choy, MD \\ Department of Orthopedic Surgery, Eulji University School of Medicine, Daejeon, Korea
}

\begin{abstract}
Purpose: Proper femoral tunnel position is important for anatomical reconstruction of the anterior cruciate ligament (ACL). The purpose of this study was to evaluate the positions of femoral and tibial tunnels created using an accessory anteromedial portal technique in single bundle ACL reconstruction.

Materials and Methods: The femoral tunnel was targeted at the mid-portion of the ACL bundles. We evaluated postoperative computed tomography scans of 32 patients treated by ACL reconstruction using a free-hand low accessory anteromedial portal technique. On the tibial side, the tunnel position was evaluated using Tsukada's method. On the femoral side, the position was evaluated using 1) the quadrant method, 2) Mochizuki's method, 3) Mochizuki's method, and 4) Takahashi's method. Tunnel obliquity was also evaluated.

Results: The mean tibial tunnel position was located at $44.6 \% \pm 2.5 \%$ anterior from the anterior margin and $48.0 \% \pm 3.0 \%$ in medial from the medial margin. The mean femoral tunnel position was located at the center between the anteromedial and posterolateral bundles: Quadrant method, $26.7 \% \pm 2.7 \% / 30.0 \% \pm 2.9 \%$; Watanabe's method, $37.7 \% \pm 2.5 \% / 26.6 \% \pm 2.2 \%$; Mochizuki's method, $38.7 \% \pm 2.7 \%$; Takahashi’s method, $21.8 \% \pm 2.2 \%$. The mean femoral tunnel obliquity was $57.7^{\circ} \pm 6.2^{\circ}$ in the sagittal plane and $49.9^{\circ} \pm 5.6^{\circ}$ in the coronal plane.

Conclusions: In anatomic single bundle ACL reconstruction, the low anteromedial portal technique can restore accurate position of the native footprint. Accurate femoral tunnel position facilitates recovery of stability and decreases graft failure rate.
\end{abstract}

Keywords: Knee, Anterior cruciate ligament reconstruction, Arthroscopy, Anteromedial portal, Computed tomography

\section{Introduction}

Single-bundle anterior cruciate ligament (ACL) reconstruction is the most common surgical intervention for ACL injuries accompanied by pain, functional impairment, and joint instability, and its efficacy has been demonstrated in a variety of studies ${ }^{1-3)}$.

Received September 6, 2013; Revised February 19, 2014;

Accepted April 10, 2014

Correspondence to: Yoon Sub Hwang, MD

Department of Orthopedic Surgery, Eulji University School of Medicine, 77 Gyeryong-ro 771beon-gil, Jung-gu, Daejeon 301-746, Korea

Tel: +82-42-611-3279, Fax: +82-42-259-1289

E-mail: poxkoreana@gmail.com

This is an Open Access article distributed under the terms of the Creative Common Attribution Non-Commercial License (http://creativecommons.org/licenses/by-nc/3.0/) which permits unrestricted non-commercial use, distribution, and reproduction in any medium, provided the original work is properly cited.
However, some recent biomechanical studies have shown that the conventional transtibial single-bundle ACL reconstruction that mainly reproduces the anteromedial bundle (AMB) is effective for restoring anterior stability but has limited impact on rotational stability provided by the posterolateral bundle (PLB) due to the more vertical orientation of the reconstructed ligament ${ }^{4)}$. The obliquity of the reconstructed ACL has been understood to have a significant influence on rotatory stability and functional recovery in the long-term. Thus, the transtibial technique that results in decreased ACL obliquity after reconstruction can be associated with negative functional outcomes ${ }^{5-7)}$.

In an attempt to circumvent the shortcomings of non-anatomical transtibial reconstruction, some authors devised anatomical ACL reconstruction techniques ${ }^{8,9)}$. Howell and Deutsch ${ }^{10)}$ suggested an outside-in technique where the femoral tunnel is drilled from the lateral side of the femur toward the medial wall of the lateral condyle. The drawbacks of the technique include in- 
consistent position of the femoral tunnel entry point depending on the direction of the guide wire and relatively high radiation exposure for drilling. Harner et al. ${ }^{11)}$ introduced an anteromedial portal technique for femoral tunneling. It allows for creation of a femoral tunnel with low obliquity contributing to improved rotatory stability but has limitations, such as, short tunnel length, poor visualization, and cortical bone destruction.

In this study, we used a low anteromedial portal for femoral tunnel placement in anatomical single-bundle ACL reconstruction to overcome disadvantages of the conventional transtibial technique. The efficacy of the accessory portal was investigated by evaluating the position and obliquity of the tibial and femoral tunnels on postoperative three-dimensional (3D) computed tomography (CT) scans.

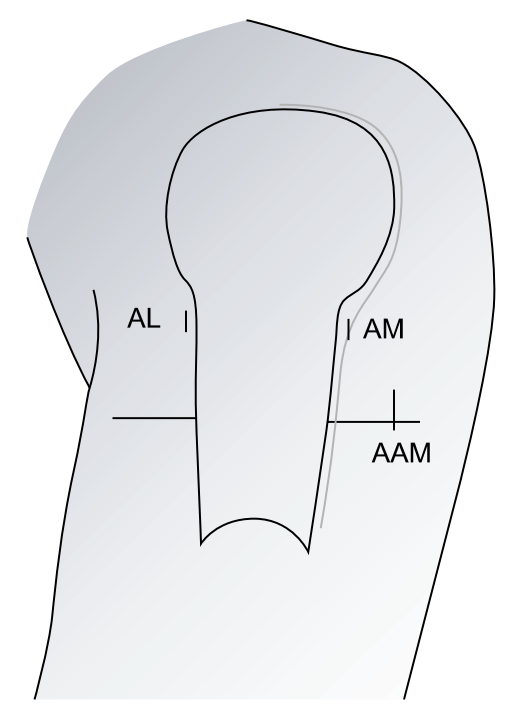

Fig. 1. Schematic drawing of three portals and their relationship to the inferior pole of the patella and borders of the patellar tendon. AL: high anterolateral portal, AM: high anteromedial portal, AAM: low accessory anteromedial portal.

\section{Materials and Methods}

\section{Patients}

A total of 53 patients underwent single-bundle ACL reconstruction using an anteromedial portal and an anterolateral portal at our institution between January 2011 and March 2012. Of these, 40 patients were available for postoperative 3D CT. Excluding 8 patients in whom the tunnel position was difficult to identify on CT scans due to periarticular fractures or previous surgery, 32 patients were included in this study. There were 26 males and 6 females with a mean age of 31.9 years (range, 16 to 54 years). The surgery was performed on the right knee in 15 and on the left knee in 17 patients. The ACL was reconstructed with an autogenous bone-patellar tendon-bone graft in 2 patients, an Achilles tendon allograft in 10 patients, and a tibialis anterior tendon allograft in 20 patients.

\section{Surgical Technique}

The femoral tunnel was created prior to the tibial tunnel via an anteromedial portal and a low anteromedial portal. The portals were established with the knee maintained at $90^{\circ}$ flexion. Under arthroscopic visualization through the anterolateral portal, an 18-gauge spinal needle was inserted as close as possible along the patellar tendon and above the medial joint line to ensure correct placement of the standard anteromedial portal. The portal was made parallel to the roof of the intercondylar notch, which was at the level or slightly above the inferior border of the patella, to prevent instrument crowding during femoral tunneling. Then, a low accessory anteromedial portal was established. After making marks on the skin that are $1 \mathrm{~cm}$ apart from each other in the medial and inferior to the anteromedial portal, using an 18-gauge spinal needle, the optimal position for the accessory portal was determined. It was located at a site that was as medial as possible

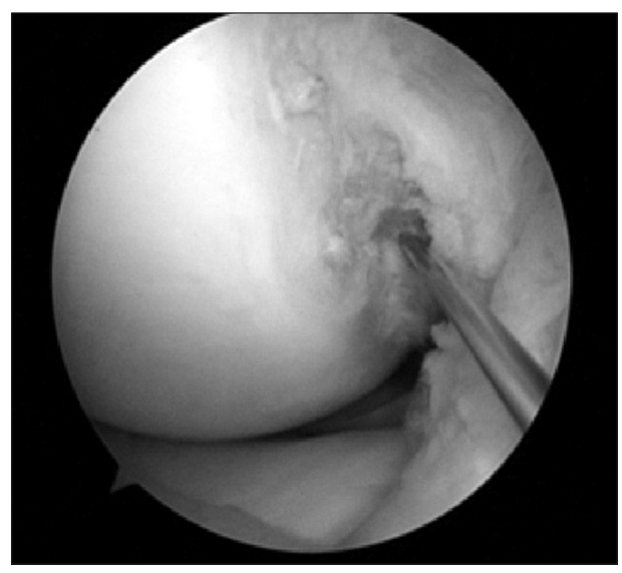

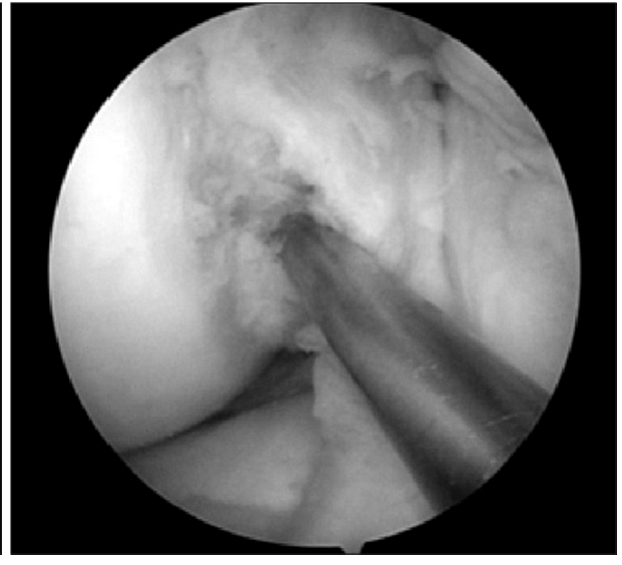

Fig. 2. Arthroscopic views through the anteromedial (AM) portal with the knee in hyperflexed position. The anterior cruciate ligament (ACL) femoral tunnel was drilled into the center of the ACL bundles through a low accessory AM portal. 

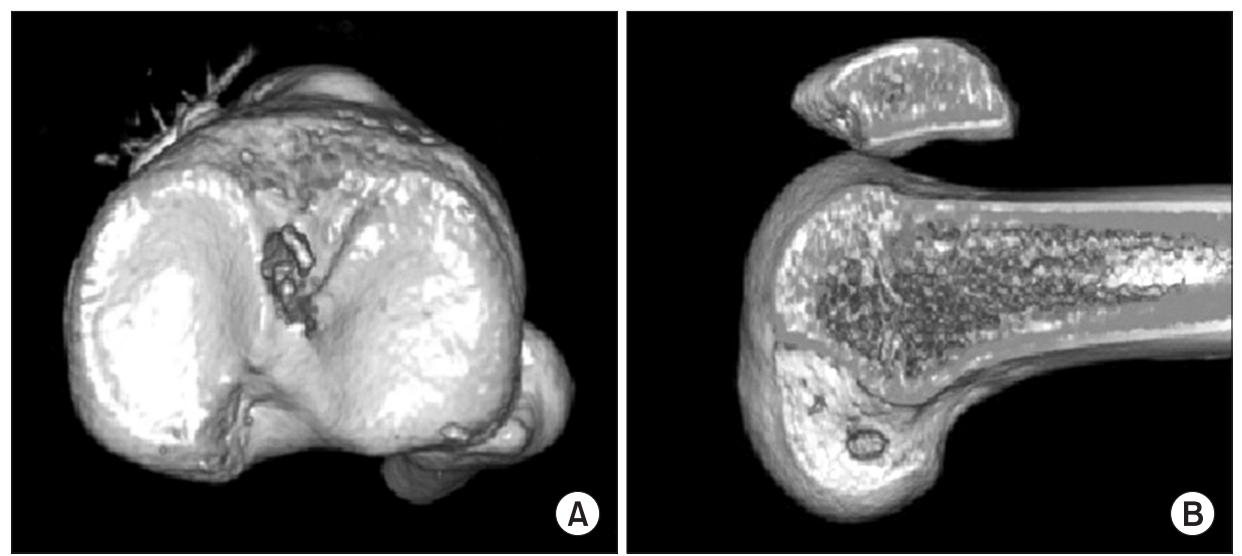

Fig. 3. Rendered three-dimensional computed tomography images of the tibia (A) and femur (B) with surrounding soft tissue removed. According to the mechanical axis, the tibia was seen from superior to inferior and the femur was seen from medial to lateral at the cut surface of the intercondylar notch.

but without the risk of damaging the medial femoral condyle by a reamer and as low as possible while avoiding the anterior horn of the medial meniscus (Fig. 1). Using the lateral intercondylar ridge as an anatomical landmark, taking care not to cause damage to the remnant fibers of the ACL on the femur, a mark was made at the center of the femoral insertion of the ACL with a free-hand technique using a microfracture awl (Chondral Pick; Arthrex Inc., Naples, FL, USA), and a guide wire was inserted through the low anteromedial portal (Fig. 2). With the knee in full flexion, the tunnel was drilled using a reamer with the same diameter of the graft. The tibial tunnel was drilled with a tibial guide set at $50^{\circ}$ angle and the tip of the aimer positioned to be $2-3 \mathrm{~mm}$ anterior to the posterior border of the anterior horn of the lateral meniscus and slightly medial to the center of the tibial attachment of the ACL. Then, the graft was passed from the tibial tunnel to the femoral tunnel and fixed. The femoral side of the graft was fixed with a metal interference screw for the bone-patellar tendonbone graft and the Achilles tendon graft and with the Endobutton fixation device (Smith \& Nephew Inc., Andover, MA, USA) for the tibialis anterior tendon allograft. For tibial tunnel fixation, a hybrid technique was used in all knees.

\section{Methods}

In all patients, CT was performed within 2 weeks after surgery with the drainage removed. After obtaining1.0mm thick crosssectional images taken perpendicular to the mechanical axis, the images were reconstructed using the Aquaris NET (TeraRecon Inc., Foster City, CA, USA) 3D rendering program to include the femur and tibia only without soft tissue. The entry points of the femoral and tibial tunnels were identified on the reconstructed scans (Fig. 3) and measurements were performed twice each by two orthopedic surgeons.

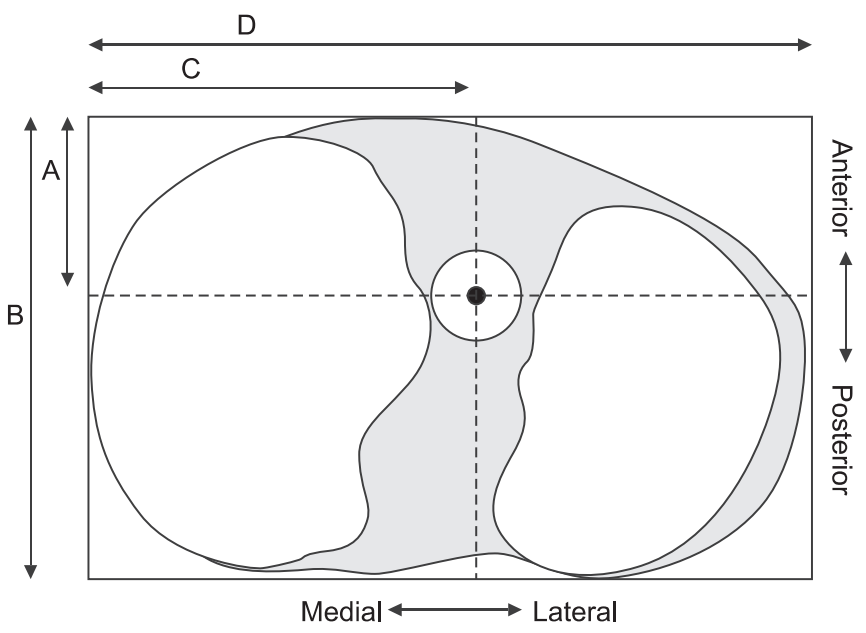

Fig. 4. Illustration of the method of Tsukada et al. ${ }^{12}$. The central point of the tibial tunnel from the anterior edge and medial edge of the tibial plateau was calculated as $\mathrm{A} / \mathrm{B}$ and $\mathrm{C} / \mathrm{D}$. A: anterior tangential line, B: lateral tangential line, C: medial tangential line, D: posterior tangential line.

\section{1) Tibial tunnel position}

The tibial tunnel position was evaluated using the method of Tsukada et al. ${ }^{12)}$ A rectangular measurement frame made up of medial tangential line (B), lateral tangential line, anterior tangential line (D), and posterior tangential line of the tibial plateau was drawn. Then, the distances from the center of the tunnel to the anterior tangential line (A) and to the medial tangential line (C) were measured. The ratios of $\mathrm{A}$ to $\mathrm{B}$ and $\mathrm{C}$ to $\mathrm{D}$ were expressed as percentage (Fig. 4).

\section{2) Femoral tunnel position}

(1) Quadrant method: According to the quadrant method suggested by Bernard et al. ${ }^{13)}$, the center of the tunnel was defined as point $\mathrm{K}$ and a rectangle was formed by the Blumensaat's line, a parallel line tangent to the most inferior margin of the lateral 


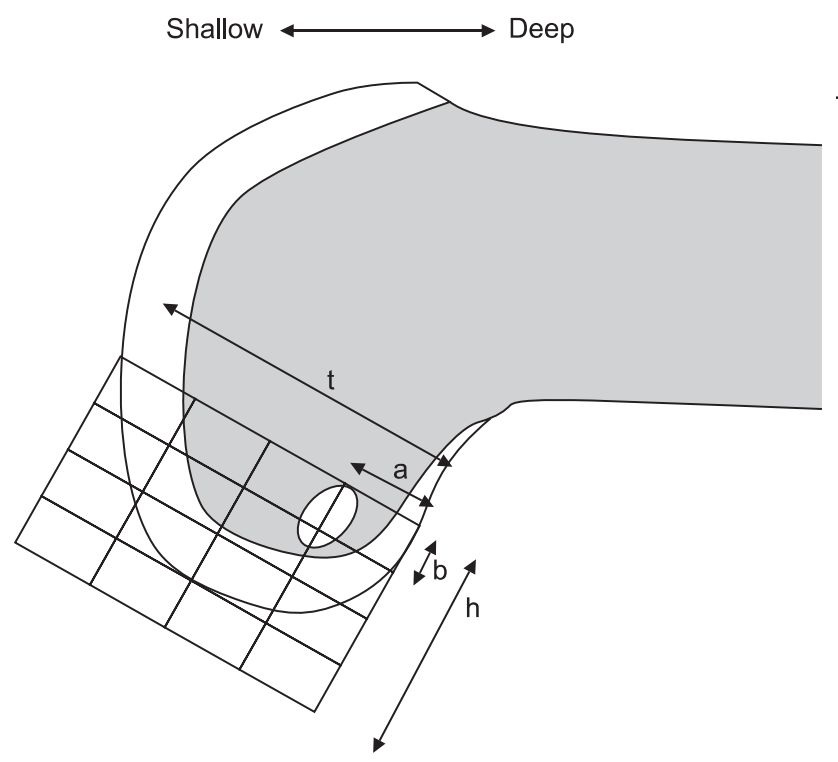

Fig. 5. Illustration of quadrant method. The central point of the femoral tunnel was calculated as $\mathrm{a} / \mathrm{t}$ and $\mathrm{b} / \mathrm{h}$. $\mathrm{t}$ : the total sagittal diameter of the lateral femoral condyle along Blumensaat's line, a: the distance of the tunnel center from the deepest subchondral contour, h: the maximum intercondylar notch height, b: the distance of the tunnel center from Blumensaat's line.
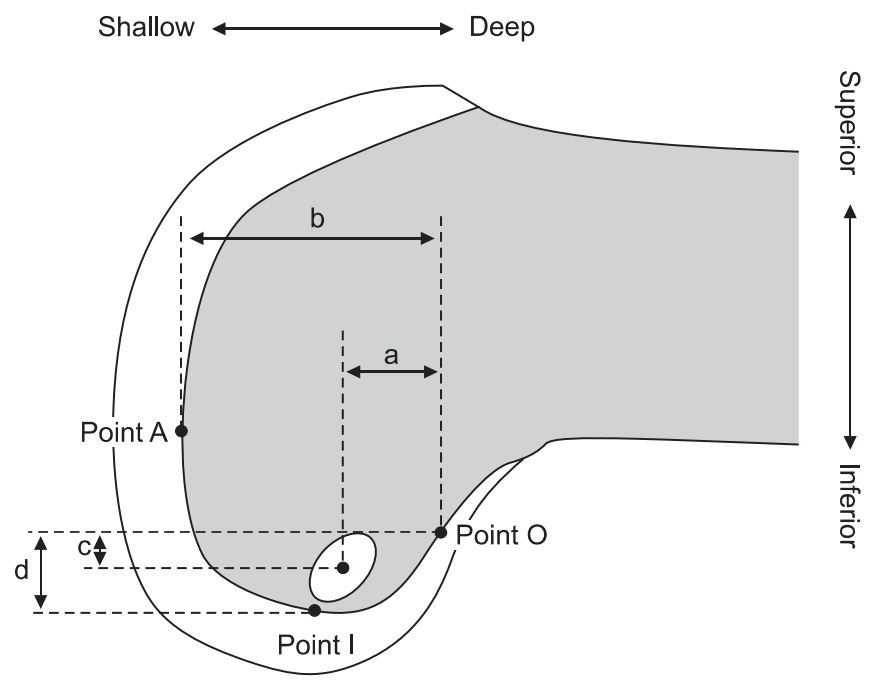

Fig. 6. Illustration of Watanabe's method. The central point of the femoral tunnel was calculated as a/b and c/d. Point O: "over-the-top position", Point A: the anterior notch outlet point, Point I: the most inferior point, a: the distance between the central point and Point $\mathrm{O}$, b: the distance between Point $\mathrm{A}$ and Point $\mathrm{O}$ along the femoral axis, $\mathrm{c}$ : the distance between the central points and Point $\mathrm{O}$, d: the distance between Point I and Point O.

condyle, and two perpendicular lines tangent to the shallowest/deepest subchondral contour of the lateral femoral condyle. Then, the following four distances were measured: the distance

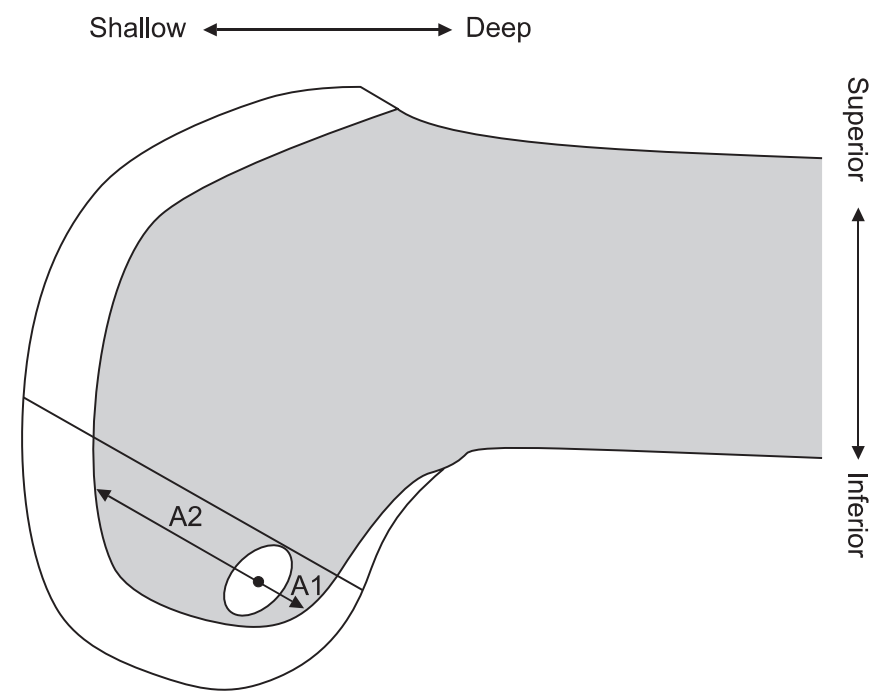

Fig. 7. Illustration of Mochizuki's method. The central point of the femoral tunnel was calculated as $\mathrm{A} 1 /(\mathrm{A} 1+\mathrm{A} 2)$. $\mathrm{A} 1$ : the distance between the deep border and the central point of the tunnel along Blumensaat's line, A2: the distance between the shallow border and the central point.
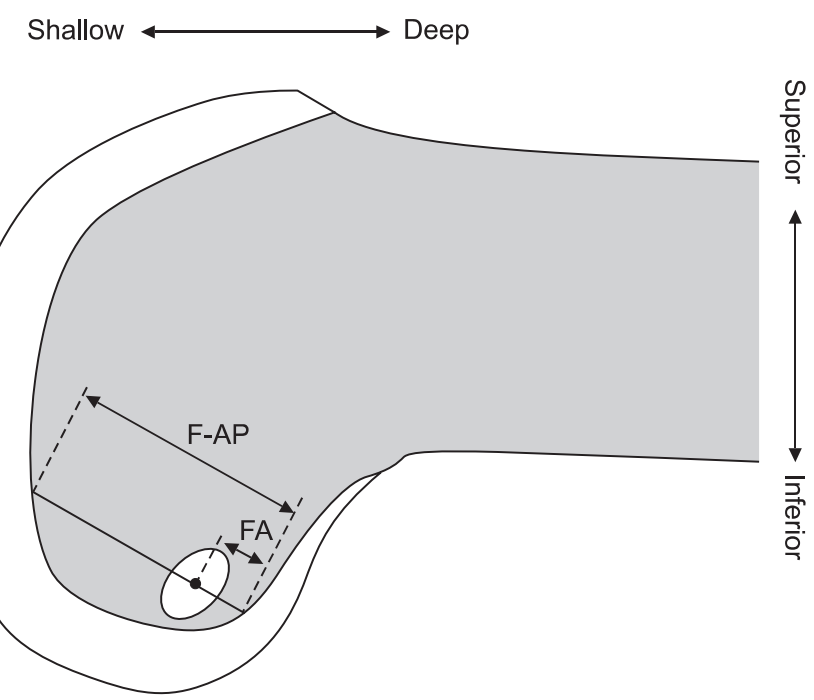

Fig. 8. Illustration of Takahashi's method. The central point of the femoral tunnel was calculated as FA/F-AP. FA: the distance between the central point of the tunnel and the deep margin of the articular surface of the lateral femoral condyle. F-AP: the distance between the shallow margin and the deep margin of the lateral femoral condyle through the central point of the tunnel.

of the lateral femoral condyle on the sagittal plane along the Blumensaat's line $(t)$, the maximum height of the intercondylar notch (h), the distance from point $\mathrm{K}$ to the deepest subchondral contour of the lateral femoral condyle (a), and the distance from point $\mathrm{K}$ to the Blumensaat's line (b). The ratios of $\mathrm{a}$ to $\mathrm{t}$ and $\mathrm{b}$ to $\mathrm{h}$ were expressed as percentage (Fig. 5). 

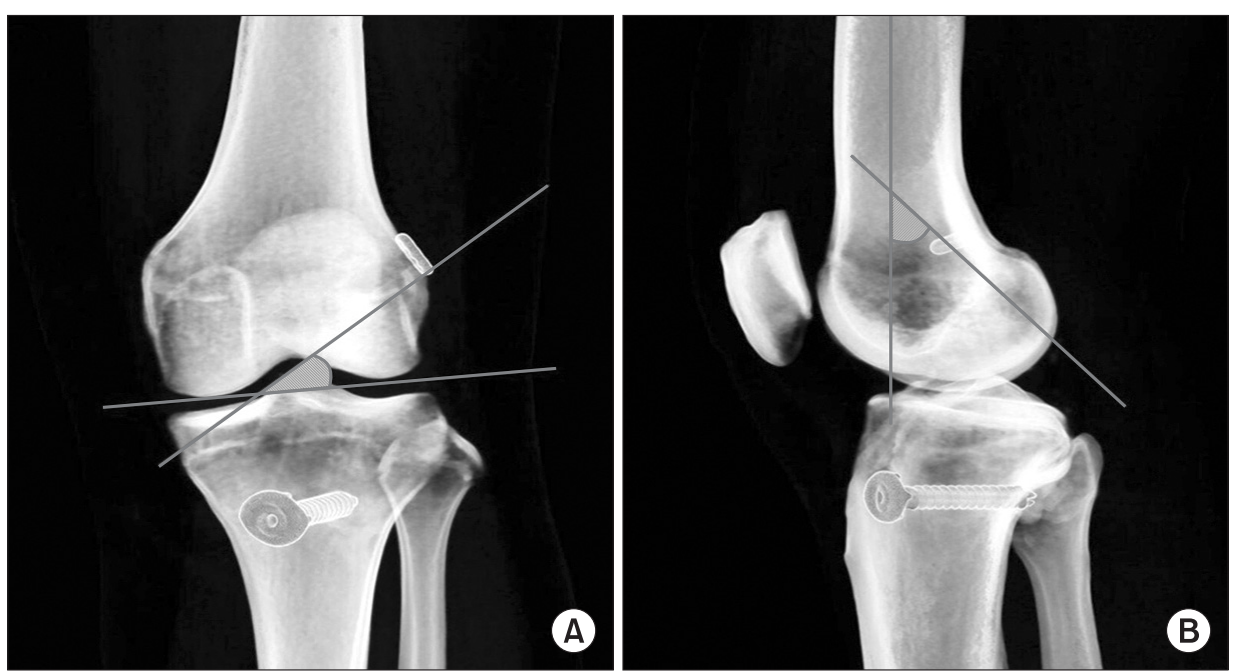

Fig. 9. (A) Coronal obliquity was measured as the angle between the femoral tunnel and a line tangent to the lateral tibial plateau. (B) Sagittal obliquity was measured as the angle between the femoral tunnel and the longitudinal axis of the femur.

(2) Watanabe method: The "over-the-top position" on the femur was defined as Point $\mathrm{O}$, the anterior notch outlet as Point A, and the most inferior point in the lateral condyle as Point I. The distance along the femoral axis between the center of the tunnel and point $\mathrm{O}$ was defined as a and between point $\mathrm{A}$ to point $\mathrm{O}$ as b. The line drawn perpendicular to the femoral axis between the tunnel center and point $\mathrm{O}$ was defined as $\mathrm{c}$ and between Point $\mathrm{I}$ and point $\mathrm{O}$ as $\mathrm{d}$. The ratios of $\mathrm{a}$ to $\mathrm{b}$ and $\mathrm{c}$ to $\mathrm{d}$ were expressed as percentage $^{14)}$ (Fig. 6).

(3) Mochizuki method: A line passing through the center of the femoral tunnel in parallel with the Blumensaat's line was drawn between the deep and shallow subchondral margins of the lateral condyle. The distance from the tunnel center to the deep margin was defined as A1 and to the shallow margin as A2. The ratio of A1 to $\mathrm{A} 1+\mathrm{A} 2$ was expressed as percentage ${ }^{15)}$ (Fig. 7).

(4) Takahashi method: The distance of a line that passes through the tunnel center between the shallow margin and the deep margin of the lateral femoral condyle was defined as F-AP. The partial distance of F-AP between the tunnel center and the margin of the articular surface of the lateral femoral condyle was defined as FA. The ratio of FA to F-AP was expressed as percentage $^{16)}$ (Fig. 8).

(5) Femoral tunnel obliquity: Coronal obliquity was measured as the angle between a line extending through the femoral tunnel and a line tangent to the lateral tibial plateau. Sagittal obliquity was measured as the angle between a line extending through the femoral tunnel and the longitudinal axis of the femur ${ }^{17)}$ (Fig. 9).

(6) Statistical analysis: SPSS ver. 12.0 (SPSS Inc., Chicago, IL, USA) was used for statistical analysis. Intraclass correlation coefficients (ICC) for intraobserver and interobserver reliability were calculated. Measurement results according to each method were analyzed.

\section{Results}

\section{Tibial Tunnel Position}

The center of the tibial tunnel was located at $44.6 \% \pm 2.5 \%$ from the anterior margin and $48.0 \% \pm 3.0 \%$ from the medial margin. In the study by Tsukada et al. ${ }^{12)}$, the AMB was located at $37.6 \% \pm 3.6 \%$ and $46.5 \% \pm 3.2 \%$, respectively and the PLB at $50.1 \% \pm 5.0 \%$ and $51.2 \% \pm 2.4 \%$, respectively.

\section{Femoral Tunnel Position}

1) Quadrant method

The femoral tunnel was located at $26.7 \% \pm 2.7 \%$ from the deepest subchondral contour of the lateral femoral condyle and at $30.0 \% \pm 2.9 \%$ from the Blumensaat's line. The values were $25 \% \pm 5 \%$ and $16 \% \pm 5 \%$, respectively, for the AMB and $29 \% \pm 6 \%$ and $42 \% \pm 3 \%$, respectively, for the PLB in the study by Yamamoto et al. ${ }^{6)}$ and $25.9 \% \pm 2.0 \%$ and $17.8 \% \pm 2.9 \%$, respectively, for the AMB and $34.8 \% \pm 2.0 \%$ and $42.1 \% \pm 3.9 \%$, respectively, for the PLB in the study by Tsukada et al. ${ }^{12)}$.

\section{2) Watanabe method}

The center of the tunnel was located at $37.7 \% \pm 2.5 \%$ from point $\mathrm{O}$ in the sagittal plane and $26.6 \% \pm 2.2 \%$ from Point $\mathrm{O}$ in the coronal plane. In the study by Tsukada et al. ${ }^{12)}$, the values were $23.7 \% \pm 3.1 \%$ and $21.9 \% \pm 5.1 \%$, respectively, for the AMB and $48.4 \% \pm 4.8 \%$ and $40.8 \% \pm 5.3 \%$, respectively, for the PLB.

\section{3) Mochizuki method}

The center of the tunnel was located at $38.7 \% \pm 2.7 \%$ from the 


\section{Lee et al. Single Bundle ACL Reconstruction by Low Accessory AM Portal Technique}

deep subchondral margin. Mochizuki et al. ${ }^{15)}$ reported the values were $28.3 \% \pm 2.1 \%$ for the AMB and $59.8 \% \pm 4.1 \%$ for the PLB, whereas the values were $25.0 \% \pm 3.9 \%$ and $45.4 \% \pm 5.5 \%$, respectively, in the study by Tsukada et al. ${ }^{12)}$

\section{4) Takahashi method}

The tunnel center was located $21.8 \% \pm 2.2 \%$ from the deep margin. Takahashi et al. ${ }^{16)}$ reported the values as $24.5 \% \pm 4.7 \%$ for the AML and $22.9 \% \pm 4.7 \%$ for the PLB. Tsukada et al. ${ }^{12)}$ reported them as $23.7 \% \pm 3.5 \%$ and $22.4 \% \pm 3.6 \%$, respectively.

\section{5) Femoral tunnel obliquity}

The coronal obliquity of the femoral tunnel was $49.9^{\circ} \pm 5.6^{\circ}$ and the sagittal obliquity was $57.7^{\circ} \pm 6.2^{\circ}$. Pascual-Garrido et al. ${ }^{18)}$ reported that the values were $50^{\circ} \pm 6^{\circ}$ and $54^{\circ} \pm 6^{\circ}$, respectively, when femoral tunneling was performed via the low anteromedial portal in ACL reconstruction using an anteromedial portal, whereas the values were $58^{\circ} \pm 9^{\circ}$ and $59^{\circ} \pm 12^{\circ}$, respectively, when a transtibial tunnel was for femoral tunneling. Bedi et al. ${ }^{17)}$ reported the values as $45.9^{\circ} \pm 6.9^{\circ}$ and $50.0^{\circ} \pm 7.5^{\circ}$, respectively, after ACL reconstruction using an anteromedial portal for femoral tunneling that was performed with the knee maintained at $120^{\circ}$ flexion.

\section{6) Statistical analysis}

Statistical analysis was performed using SPSS ver. 12.0 (SPSS Inc., Chicago, IL, USA) with a significance level of $\mathrm{p}<0.05$. The ICC for interobserver reproducibility was $0.82-0.96$ and the ICC for intraobserver reproducibility was $0.88-0.98$.

\section{Discussion}

In this study, the tibial and femoral tunnel position and obliquity were evaluated with 3D CT after single-bundle ACL reconstruction using a low accessory anteromedial portal. The tibial and femoral tunnels were consistently located between the AMB and PLB and the tunnel obliquity was low. No complications associated with tunneling, such as posterior cortical bone destruction, were noted on CT scans.

The obliquity and anatomical placement of the femoral tunnel has been a major point of interest in recent research on ACL reconstruction. More vertical tunnel orientation has been correlated with rotatory instability, and nonanatomical tunnel placement has been associated with pain and instability, which could eventually result in poor outcomes and graft rupture ${ }^{19,20)}$. Kaseta et al. ${ }^{20)}$ emphasized the importance of portal position and reconstruction surgical technique, which determines the femoral tunnel position and ultimately affects the outcomes of ACL reconstruction.

The conventional transtibial technique where the tibial tunnel is created first does not allow for anatomical femoral tunnel placement. The relatively high position of the femoral tunnel requires close to perpendicular placement of a graft, which is not conducive to restoration of rotatory stability.

Modified transtibial techniques were designed to overcome the shortcomings of the traditional transtibial technique by allowing changes in the tibial tunnel position and the insertion site of a reamer ${ }^{10,21)}$ for less oblique femoral tunnel placement. However, the techniques have inherent problems: the tunnel orientation is still more oblique than that in other surgical techniques, the acceptable margin of error is narrow, and the tibial tunnel should be located through the medial collateral ligament and the medial condyle or at an excessively posterior site. In a cadaver study by Sim et al. ${ }^{22)}$, a modified transtibial technique was less effective than the anteromedial portal technique or outside-in technique in restoring anterior translation at $0^{\circ}$ flexion of the knee.

With an outside-in technique, a drilling pin is introduced from a lateral site toward a target point determined via the anteromedial portal. This technique facilitates creation of a femoral tunnel with low obliquity, but there is a relatively high risk of misplacement depending on the drilling wire insertion site and drilling angle. The employment of fluoroscopy for overcoming this problem increases radiation exposure. In addition, notchplasty is often necessitated to ensure visualization and guide wire insertion.

The conventional anteromedial portal allows for less oblique femoral tunnel orientation compared to the transtibial technique. However, the field of view is limited by the sole availability of the anterolateral portal for arthroscopic visualization, extensive cortical bone destruction is unavoidable, and the femoral tunnel is relatively short compared to that in modified transitibial techniques due to the insufficient working space and angle ${ }^{11,23)}$. Further, intraoperative visualization requires an additional procedure of removing tunneling instrument and transferring the arthroscope, which increases the risk of enlarging the tunnel diameter and changing the tunnel obliquity.

The accessory anteromedial portal technique can be an alternative to the abovementioned techniques that offers the following $\operatorname{advantages}^{24)}$ :

1. Femoral tunneling can be performed without interference with visualization by the lateral femoral condyle by using the anteromedial portal as a viewing portal. Tunnel position can be identified intraoperatively without drilling instrument removal.

2. The femoral tunnel can be created close to the lateral wall of 
the notch by adjusting the obliquity compared to the anteromedial technique, which allows more flexibility with tunnel obliquity and length according to bone quality, graft type, and graft fixation method.

3. Notchplasty for working space and visualization can be avoided.

The free-hand drilling technique used in this study makes the most of the advantages of the accessory anteromedial portal technique in ACL reconstruction, offering sufficient working space and flexibility with tunnel position and obliquity. However, one pitfall of the techniques is that inaccurate tunnel position planning can lead to inconsistent tunnel placement, resulting in improper tunnel obliquity and posterior cortical bone destruction. To avoid these problems, we created a low accessory anteromedial portal in the lateral knee and placed the femoral tunnel at the center between the AMB and PLB footprints to obtain consistently femoral tunnels with low obliquity and avoid cortical bone destruction.

Currently, the research on ACL reconstruction is taking a biomechanical approach. Efforts are focused on determining the femoral tunnel entry point based on the ACL footprints and securing longitudinal stability and proper fixation strength based on the assessment of the femoral tunnel obliquity and length. The majority of studies employed plain radiography or fluoroscopy for postoperative tunnel evaluation in human subjects or cadavers. Meuffels et al. ${ }^{25)}$ compared the efficacy of plain radiography, $\mathrm{CT}$, and 3D virtual reality imaging technique for the assessment of postoperative tunnel position. In the study, the CT scans and $3 \mathrm{D}$ virtual reality images were found to be more reliable than radiographs: the interobserver agreement and intraobserver agreement were higher for the former two techniques. The femoral tunnel could be identified in $52 \%-82 \%$ of the cases on plain radiographs. Obtaining lateral radiographs was challenging in

Table 1. Central Point of the Anterior Cruciate Ligament Footprint by Quadrant Method

\begin{tabular}{lcc}
\hline \multicolumn{1}{c}{ Article } & Mean depth (\%) & Mean height (\%) \\
\hline Tsukada et al. $^{12)}$ & 30.35 & 29.95 \\
Yamamoto et al. $^{6)}$ & 27 & 29 \\
Steckel et al. $^{26)}$ & 26.9 & 27.5 \\
Colombet et al. $^{27)}$ & 29.35 & 36.45 \\
Zantop et al. $^{28)}$ & 23.9 & 37.95 \\
Guo et al. $^{29)}$ & 43.1 & 38.3 \\
Mean of means & 30.1 & 33.19 \\
Our study & 26.6 & 30 \\
\hline
\end{tabular}

terms of technicality and time, and the risk of compromising the accuracy of assessment was higher with radiography than with CT.

Quadrant method is the most common among various methods to assess femoral tunnel position. Assuming the center of the ACL footprints is located in the middle between the AMB and the PLB, Tsukada et al. ${ }^{12)}$ reported the center of the ACL footprints was located at $30.35 \%$ from the deep margin and $29.95 \%$ from the Blumensaat's line. The values were $27 \%$ and $29 \%$, respectively, according to Yamamoto et al. ${ }^{6}, 26.9 \%$ and $27.5 \%$, respectively, according to Steckel et al. ${ }^{26)}, 29.35 \%$ and $36.45 \%$, respectively, according to Colombet et al. ${ }^{27)}, 23.9 \%$ and $37.95 \%$, respectively, according to Zantop et al. ${ }^{28)}$, and $43.1 \%$ and $38.3 \%$, respectively, according to Guo et al. ${ }^{29)}$ (Table 1). In our study, the values were $26.6 \%$ and $30 \%$, respectively, which was similar to the mean values of other studies, but the distance from the Blumensaat's line was slightly greater than the mean values in the literature. This can be attributed to our effort to avoid creating a tunnel with excessively low obliquity to prevent tunnel shortening inherent to the anteromedial technique and secure sufficient tunnel length for Endobutton fixation.

The limitations of this retrospective study on the tunnel position after ACL reconstruction using a low anteromedial portal include: 1) there was no comparison with the transtibial portal technique, 2) clinical indicators, such as functional scores and stability, were not addressed; 3) the center of the original insertion site of the ACL could not be measured because the study involved human subjects; thus, comparisons with the tibial and femoral tunnel measurement values in previous studies were made, and 4) the influence of the angle between the femoral tunnel and the graft on reconstruction was not taken into consideration, which we believe should be addressed in further studies.

However, the significance of this study is that it is one of the few studies on ACL reconstruction using a free-hand technique with an accessory anteromedial portal and the evaluation of the postoperative tunnel position and obliquity.

\section{Conclusions}

ACL reconstruction using a free-hand technique with a low accessory anteromedial portal consistently allowed femoral and tibial tunnel placement at the center of the ACL footprints and with low obliquity. The postoperative tunnel position was identified with 3D CT in all knees and the ICC for interobserver and intraobserver reliability was high. Precise femoral tunnel placement has significant influence on restoration of anteroposterior 
and rotatory stability and reduction of graft failure.

\section{Conflict of Interest}

No potential conflict of interest relevant to this article was reported.

\section{References}

1. Buss DD, Warren RF, Wickiewicz TL, Galinat BJ, Panariello R. Arthroscopically assisted reconstruction of the anterior cruciate ligament with use of autogenous patellar-ligament grafts. Results after twenty-four to forty-two months. J Bone Joint Surg Am. 1993;75:1346-55.

2. Feller JA, Webster KE. A randomized comparison of patellar tendon and hamstring tendon anterior cruciate ligament reconstruction. Am J Sports Med. 2003;31:564-73.

3. Shelbourne KD, Nitz P. Accelerated rehabilitation after anterior cruciate ligament reconstruction. Am J Sports Med. 1990;18:292-9.

4. Lim HC, Yoon YC, Wang JH, Bae JH. Anatomical versus non-anatomical single bundle anterior cruciate ligament reconstruction: a cadaveric study of comparison of knee stability. Clin Orthop Surg. 2012;4:249-55.

5. Bowers AL, Bedi A, Lipman JD, Potter HG, Rodeo SA, Pearle AD, Warren RF, Altchek DW. Comparison of anterior cruciate ligament tunnel position and graft obliquity with transtibial and anteromedial portal femoral tunnel reaming techniques using high-resolution magnetic resonance imaging. Arthroscopy. 2011;27:1511-22.

6. Yamamoto Y, Hsu WH, Woo SL, Van Scyoc AH, Takakura Y, Debski RE. Knee stability and graft function after anterior cruciate ligament reconstruction: a comparison of a lateral and an anatomical femoral tunnel placement. Am J Sports Med. 2004;32:1825-32.

7. Lee MC, Seong SC, Lee S, Chang CB, Park YK, Jo H, Kim $\mathrm{CH}$. Vertical femoral tunnel placement results in rotational knee laxity after anterior cruciate ligament reconstruction. Arthroscopy. 2007;23:771-8.

8. van Eck CF, Lesniak BP, Schreiber VM, Fu FH. Anatomic single- and double-bundle anterior cruciate ligament reconstruction flowchart. Arthroscopy. 2010;26:258-68.

9. Yagi M, Wong EK, Kanamori A, Debski RE, Fu FH, Woo SL. Biomechanical analysis of an anatomic anterior cruciate ligament reconstruction. Am J Sports Med. 2002;30:660-6.

10. Howell SM, Deutsch ML. Comparison of endoscopic and two-incision techniques for reconstructing a torn anterior cruciate ligament using hamstring tendons. Arthroscopy. 1999;15:594-606.

11. Harner CD, Honkamp NJ, Ranawat AS. Anteromedial portal technique for creating the anterior cruciate ligament femoral tunnel. Arthroscopy. 2008;24:113-5.

12. Tsukada H, Ishibashi Y, Tsuda E, Fukuda A, Toh S. Anatomical analysis of the anterior cruciate ligament femoral and tibial footprints. J Orthop Sci. 2008;13:122-9.

13. Bernard M, Hertel P, Hornung H, Cierpinski T. Femoral insertion of the ACL: radiographic quadrant method. Am J Knee Surg. 1997;10:14-21.

14. Watanabe S, Satoh T, Sobue T, Koga Y, Oomori G, Nemoto A. Three dimensional evaluation of femoral tunnel position in anterior cruciate ligament reconstruction. Hiza J Japan Knee Soc. 2005;30:253-6.

15. Mochizuki T, Muneta T, Nagase T, Shirasawa S, Akita KI, Sekiya I. Cadaveric knee observation study for describing anatomic femoral tunnel placement for two-bundle anterior cruciate ligament reconstruction. Arthroscopy. 2006;22:35661.

16. Takahashi M, Doi M, Abe M, Suzuki D, Nagano A. Anatomical study of the femoral and tibial insertions of the anteromedial and posterolateral bundles of human anterior cruciate ligament. Am J Sports Med. 2006;34:787-92.

17. Bedi A, Raphael B, Maderazo A, Pavlov H, Williams RJ 3rd. Transtibial versus anteromedial portal drilling for anterior cruciate ligament reconstruction: a cadaveric study of femoral tunnel length and obliquity. Arthroscopy. 2010;26:34250.

18. Pascual-Garrido C, Swanson BL, Swanson KE. Transtibial versus low anteromedial portal drilling for anterior cruciate ligament reconstruction: a radiographic study of femoral tunnel position. Knee Surg Sports Traumatol Arthrosc. 2013;21:846-50.

19. Giron F, Buzzi R, Aglietti P. Femoral tunnel position in anterior cruciate ligament reconstruction using three techniques: a cadaver study. Arthroscopy. 1999;15:750-6.

20. Kaseta MK, DeFrate LE, Charnock BL, Sullivan RT, Garrett WE Jr. Reconstruction technique affects femoral tunnel placement in ACL reconstruction. Clin Orthop Relat Res. 2008;466:1467-74.

21. Kondo E, Yasuda K, Ichiyama H, Azuma C, Tohyama H. Radiologic evaluation of femoral and tibial tunnels created with the transtibial tunnel technique for anatomic double-bundle anterior cruciate ligament reconstruction. Arthroscopy. 
2007;23:869-76.

22. Sim JA, Gadikota HR, Li JS, Li G, Gill TJ. Biomechanical evaluation of knee joint laxities and graft forces after anterior cruciate ligament reconstruction by anteromedial portal, outside-in, and transtibial techniques. Am J Sports Med. 2011;39:2604-10.

23. Chang CB, Choi JY, Koh IJ, Lee KJ, Lee KH, Kim TK. Comparisons of femoral tunnel position and length in anterior cruciate ligament reconstruction: modified transtibial versus anteromedial portal techniques. Arthroscopy. 2011;27:138994.

24. Brown $\mathrm{CH}$ Jr, Spalding T, Robb C. Medial portal technique for single-bundle anatomical anterior cruciate ligament (ACL) reconstruction. Int Orthop. 2013;37:253-69.

25. Meuffels DE, Potters JW, Koning AH, Brown CH Jr, Verhaar JA, Reijman M. Visualization of postoperative anterior cruciate ligament reconstruction bone tunnels: reliability of standard radiographs, CT scans, and 3D virtual reality im- ages. Acta Orthop. 2011;82:699-703.

26. Steckel H, Musahl V, Fu FH. The femoral insertions of the anteromedial and posterolateral bundles of the anterior cruciate ligament: a radiographic evaluation. Knee Surg Sports Traumatol Arthrosc. 2010;18:52-5.

27. Colombet P, Robinson J, Christel P, Franceschi JP, Djian P, Bellier G, Sbihi A. Morphology of anterior cruciate ligament attachments for anatomic reconstruction: a cadaveric dissection and radiographic study. Arthroscopy. 2006;22:984-92.

28. Zantop T, Wellmann M, Fu FH, Petersen W. Tunnel positioning of anteromedial and posterolateral bundles in anatomic anterior cruciate ligament reconstruction: anatomic and radiographic findings. Am J Sports Med. 2008;36:65-72.

29. Guo L, Yang L, Wang AM, Wang XY, Dai G. Roentgenographic measurement study for locating femoral insertion site of anterior cruciate ligament: a cadaveric study with XCaliper. Int Orthop. 2009;33:133-7. 